\title{
Clasificación y análisis de páginas web comerciales extremeñas
}

\author{
M. ${ }^{a}$ del Rosario Fernández Falero

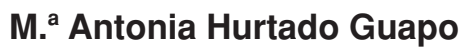 \\ Diego Peral Pacheco \\ Universidad de Extremadura (España)
}

\section{Resumen}

El objeto de esta comunicación es la clasificación y análisis de páginas web de empresas de Extremadura dedicadas al comercio electrónico, así como la evolución de las mismas. Para ello se han realizado búsquedas en la Red mediante buscadores (Google, Altavista) con las palabras clave empresas extremeñas, empresas extremeñas en red, Extremadura en red, Extremadura on line, web extremeñas en red. Los datos obtenidos fueron entre otros una serie de páginas web de empresas extremeñas, algunas de ellas directorios de empresas de esta comunidad en Internet. Los resultados muestran qué sectores comerciales extremeños se están estableciendo en la Red. Al observar la evolución a través del tiempo, podemos ver qué sectores se están consolidando, cuáles van aumentando, cuáles van disminuyendo y cuáles desaparecen. El análisis aplicado a cada página web no solo sirve para determinar a qué sector comercial pertenece dicha página, sino también para establecer el tipo de venta que realiza: así, se estudia qué páginas son de venta directa y cuáles de venta indirecta, y se clasifican. En un principio en nuestro trabajo se ordenaban con una clasificación establecida en una de las páginas web consultadas pero, dado que esta clasificación presentaba a nuestro juicio algunos errores, nos propusimos clasificarlas siguiendo la Clasificación Decimal Universal. Creemos que la CDU se adapta a las características propias de las páginas web y por ello la proponemos como clasificación para las mismas, no solo en el caso de Extremadura sino en toda la Red. El comercio electrónico es, por lo que podemos observar cotidianamente y que confirmamos en nuestro estudio, una nueva forma de hacer negocios, donde los objetivos son los mismos que rigen en el mercado tradicional: ventas y rentabilidad. La diferencia está en la flexibilidad y la capacidad de reacción que tengan las empresas en este nuevo entorno. Podemos observar en el estudio que existe una gran diferencia entre el número de páginas web de venta indirecta y el de las de venta directa, y cómo se está produciendo un aumento del número de estas últimas, merced sobre todo a un conjunto de sectores en los que se está produciendo el mayor incremento. El impacto del comercio electrónico en

Scire. $12: 1$ (en.-jun. 2006) 163-178. ISSN 1135-3761. 
Extremadura tiene una serie de características propias que muestran la importancia de estudiar la Red localmente: en general, podemos destacar que sectores como automoción, hostelería, turismo, comunicación e informática, y algunos otros cumplen las previsiones de crecimiento a nivel nacional; sin embargo, a diferencia de lo que ocurre en el resto de comunidades autónomas, la alimentación es uno de los sectores más presentes en la Red, mientras que el sector financiero y los seguros, que están entre los que muestran mayores posibilidades de crecimiento a nivel nacional, en Extremadura se encuentran entre los de menor presencia.

Palabras clave: Comercio electrónico. Extremadura. Web comerciales.

\section{Abstract}

The aim of this work is the classification and analysis of websites of Extremaduran companies dealing with the electronic commerce, as well as their evolution. For this, searches have been carried out in Internet by means of search engines (Google, Altavista), using the keywords empresas extremeñas, empresas extremeñas en red, Extremadura en red, Extremadura on line, web extremeñas en red. The result was, among others, a series of web pages of Extremaduran companies. and some of them were directories of companies of this region in Internet. The results show what commercial Extremaduran sectors are established in the Web. Having observed the evolution across the time, we can see what sectors are consolidated, which ones are expanding, which ones are decreasing and which ones disappear. The analysis applied to every website is not only useful for determining what commercial sector that site belongs to, but also for establishing the kind of sale carried out through it: this way, direct and indirect sale websites are differenciated and classified. At first, they were arranged in our work according to a classification set in one of the websites we consulted, but, as we thought that this classification had some mistakes, we suggested following the UDC, which is suited to the features of the websites; this is why we suggested it as classification system, not only for Extremadura but for the whole Web. The electronic commerce is, according to what we see everyday and is confirmed by our study, a new way of business where the aims are the same that those of the traditional trade: sales and profitability. The difference is the flexibility and capacity to react of the companies in this new environment. We can see that there is a great difference between the amount of indirect sale websites and those of direct sale, which are rising thanks, above all, to some sectors in which the major increase is taking place. The impact of the electronic commerce in Extremadura has some features that show the importance of studying the Web locally: in general, we can emphasize that sectors as automotive, hotels and tourism, communication and computer science, and some others achieve the national growth forecasts; nevertheless, unlike what it happens in the rest of autonomous regions, food is one of the more usually found sectors 
in the Web, whereas the financial and insurance sectors, which are among the ones with most possibilities of growth in the country, are hardly ever found in Extremadura.

Keywords: Electronic commerce. Extremadura. Commercial websites.

\section{Introducción}

Las empresas extremeñas, siguiendo la estela de otras de ámbito nacional, han iniciado su actividad comercial en la Red. Con este trabajo analizamos a lo largo de aproximadamente tres años cómo ha sido tal acceso comercial a este nuevo medio. Así, en un principio los empresarios (Santamaría y Escobar, 2004) elaboran su página web más como una moda que como una estrategia comercial; por eso al principio la mayoría de sitios web son páginas que contienen cierta información sobre la empresa y cuya dirección el empresario imprime en los folletos comerciales e incluso en los vehículos de reparto, es decir, es otra forma de hacer publicidad. Pero poco a poco el empresario empieza a darse cuenta de las posibilidades de Internet y de que esas páginas con información comercial pueden ir a más.

En esta segunda etapa del comercio electrónico, las empresas de Internet se muestran ante todo como lo que son: empresas cuya finalidad es hacer negocio; lo único que cambia es el medio, y adaptarse a ese medio y generar estrategias que faciliten la consecución de sus objetivos empresariales se convierte en el fin que persigue el comercio electrónico.

De manera que si consideramos que el comercio electrónico es, como lo definen Félix Santamaría y Modesto Escobar (004), "Cualquier actividad en la Red realizada con ánimo comercial, que pueda generar la realización de una transacción comercial, tanto en la web como fuera de ella, en el mismo instante o más tarde", de esta definición se desprende que el comercio electrónico abarca toda la actividad comercial de la Red, aunque en realidad en el vocabulario comercial (eMarket Services, 2005) se establecen una series de distinciones, tales como:

- e-commerce, o sea, 'comercio electrónico', donde se compra y vende en línea: consiste en la interacción entre cliente y vendedor y la transacción al pagar el producto.

- e-bussines, que se puede traducir como 'negocio electrónico': la Red se usa para obtener un beneficio de ella; este término engloba el comercio electrónico y además incluye toda la parte de administración, publicidad, servicio de atención al cliente, etcétera; en definitiva, toda gestión de la empresa en la Red.

- e-marketplaces, o sea, 'mercado electrónico', que se puede describir como una tienda virtual donde los vendedores, suministradores, distribuidores y vendedores encuentran e intercambian información, colaboraciones entre ellos para integrarse en determinados portales, etcétera. En definitiva este

Scire. $12: 1$ (en.-jun. 2006) 163-178. ISSN 1135-3761. 
concepto forma parte del negocio electrónico si este intercambio de información tiene como finalidad realizar alguna operación comercial, y es comercio electrónico cuando solamente intercambian información sobre las características de las transacciones que vayan a realizar.

Uno de los principales negocios de la Red es la venta del producto, de manera que la página web ha pasado de solo publicitar a vender los productos, como por ejemplo en el caso de una empresa dedicada a la venta de software: con solo hacer click con el ratón, el usuario puede solicitar el producto, pagarlo y obtenerlo a través de la Red sin moverse de casa, y si tenemos en cuenta, como indica un informe de la Asociación Española de Comercio Electrónico (AECE, 2003), que la comodidad es el primer factor que señalan los usuarios de Internet para su uso comercial, este tipo de productos que se obtienen a través de la Red son los más demandados; pero cabe la posibilidad de solicitar el producto y que nos lo envíen a nuestro domicilio, tipo de transacción que también tiene éxito en Internet.

En lo que se refiere a cuál es el producto que mejor se vende por Internet, señalaremos que es aquel que se adapta mejor a las características de la misma, en concreto aquel que reúna alguna de las características que indica un informe de Andersen Consulting y el Banco Santander (Andersen, 2000):

Productos fáciles de distribuir o intangibles.

Productos que no se prueban antes de comprar.

Productos que no satisfacen necesidades inmediatas.

Productos que se consumen fuera del lugar de compra.

Productos en los que prima el contenido sobre la apariencia.

Productos de marcas conocidas.

Productos caros (el coste de distribución es un pequeño porcentaje del precio).

Productos complejos que requieren mucha información.

Productos sensibles al precio.

Productos con poca diferenciación entre distintos oferentes.

Productos en los que la privacidad del adquirente facilite la compra.

Con estas características, vemos que los productos financieros, empresas de consultorías o seguros son los que mejor se adaptan a este medio, y así es en general en España, excepto en Extremadura, donde como demuestra este trabajo vamos a ver que el comercio electrónico tiene características propias, fruto quizás de la situación empresarial extremeña.

\section{Material y método}

Los resultados se han obtenido realizando en primer lugar una búsqueda en la Red mediante los buscadores Google y Altavista, con las palabras clave empresas extremeñas, empresas extremeñas en red, Extremadura en red, Extremadura on line, web extremeñas en red. 
Con las palabras clave empresas extremeñas se recuperó la página web www.extremaduraempresas.com, en la que venía una relación de empresas extremeñas en la red, localizándose el resto en la página www.masempresa.com, que es un portal de empresas y profesionales. Una vez realizada la búsqueda, los resultados obtenidos se estudian siguiendo los pasos que se detallan a continuación.

Se determina si son páginas comerciales y se eliminan aquellas en las que algunas empresas aparecen en fichas como parte de una página web donde no se añade información de la empresa.

Se procede al análisis de las mismas estudiando los productos en venta y la forma de venta de los mismos, directa o indirecta, teniendo en cuenta que se considera venta directa cuando desde el ordenador (apretando una tecla) se puede realizar la operación de comprar el producto y este es enviado donde el usuario lo solicite, mientras que en la compra indirecta se remite al comprador a algún centro comercial (solo accede a la información sobre el producto pero no puede comprarlo).

Analizamos las páginas para determinar qué tipo de producto se vende y organizamos las mismas con la clasificación que utiliza la página web www.extremaduraempresas.com (dentro de esta clasificación, en el apartado nominado como "otros", se han incluido una serie de páginas web que muestran unos contenidos notablemente diversos que hacen difícil su asignación a los diferentes apartados que se han establecido).

En esta ocasión hemos realizado la búsqueda, conteo y análisis de las páginas web comerciales en tres ocasiones. La primera búsqueda la realizamos en octubre del año 2003, la siguiente en octubre de 2004 y la última en abril de 2005.

Una vez obtenidos los datos observamos que la clasificación no se ajusta bien a la naturaleza de los datos recogidos y optamos por utilizar otra forma de ordenarlos, basándonos para ello en trabajos anteriores (Caro, 1998): decidimos utilizar la Clasificación Decimal Universal y obtuvimos los resultados que se muestran a continuación.

\section{Resultados}

Los resultados se muestran en tablas. En primer lugar, la tabla I contiene los datos en orden descendente según el código que le corresponde al aplicar la CDU.

Para poder hacer un estudio comparativo de estos tres años, hemos tenido que reunir algunos datos en lo que llamamos sectores (que aparecen en negrita). Esto es debido a que cuando recuperamos los datos de 2003 partimos de una clasificación elaborada por nosotros que organizaba las páginas web comerciales en sectores y subsectores; por ello, al utilizar la CDU hemos mantenido esa división. Así, por ejemplo, el sector de hostelería y turismo suma los datos que se clasificaron como tales y los siguientes subsectores: hostales y pensiones, hoteles, turismo rural,

Scire. $12: 1$ (en.-jun. 2006) 163-178. ISSN 1135-3761. 
camping, viajes/agencias. El resto de datos corresponden a los resultados obtenidos al hacer las búsquedas de páginas web comerciales extremeñas durante octubre de 2004 y abril de 2005 .

\begin{tabular}{|c|c|c|c|c|}
\hline Sector & CDU & 2003 & 2004 & 2005 \\
\hline Lectura & 28 & 34 & 37 & 23 \\
\hline Comercio del suelo. Comercio inmobiliario & 332.7 & 56 & 74 & 62 \\
\hline Mercado de las viviendas de alquiler & 339.187 .6 & 23 & 19 & 24 \\
\hline Finanzas. Finanzas públicas. Banca. Moneda. Bolsa & 336 & 101 & 142 & 125 \\
\hline Hospitales & 364.444 & & 7 & 5 \\
\hline Seguros & 368 & 78 & 135 & 120 \\
\hline Clases de empresas de seguros & 368.03 & 60 & 119 & 102 \\
\hline Educación. Enseñanzas. Formación & 37 & 74 & 78 & 48 \\
\hline $\begin{array}{l}\text { Formación general (formación) + otros métodos } \\
\text { de enseñanza (academias) }\end{array}$ & $370.31+371.39$ & 74 & 78 & 48 \\
\hline Hostelería y turismo & 379.8 & 615 & 817 & 664 \\
\hline Hostales y pensiones & 379.832 & 297 & 312 & 284 \\
\hline Hoteles & 379.832 & 132 & 167 & 131 \\
\hline Turismo rural & 379.834 & 133 & 266 & 189 \\
\hline Camping & 379.837 & 28 & 33 & 26 \\
\hline Viajes/agencias & $379.85+656.076$ & 25 & 39 & 34 \\
\hline Fiestas nacionales. Festejos populares & 394.2 & 4 & 14 & 13 \\
\hline Ciencia y tecnología de los ordenadores & 4 & 10 & 60 & 110 \\
\hline Protección del medio ambiente & 504.06 & 8 & 10 & 10 \\
\hline Química & 54 & 9 & 7 & 6 \\
\hline $\begin{array}{l}\text { Dietética. Principios nutricionales aplicados } \\
\text { a las bebidas y a los alimentos }\end{array}$ & 613.2 & 237 & 274 & 287 \\
\hline Jamones y embutidos & 613.8 & 56 & 70 & 70 \\
\hline Bebidas y bodegas & 613.663 .28 & 25 & 39 & 40 \\
\hline Almazaras y aceitunas & 665.327 & 34 & 61 & 62 \\
\hline Automoción & 629 & 92 & 147 & 91 \\
\hline Taller & 629.083 & 40 & 59 & 38 \\
\hline Recambio & 629.084 & 18 & 27 & 13 \\
\hline Ventas & 629.167 & 34 & 55 & 35 \\
\hline Higiene y salud pública & 614 & 52 & 72 & 72 \\
\hline Farmacia general y profesional & 615.1 & & 8 & 4 \\
\hline Ingenierías & 62 & 76 & 112 & 73 \\
\hline Ingeniería eléctrica & 621.3 & 40 & 60 & 37 \\
\hline Electrodomésticos & 621.313 & 18 & 23 & 14 \\
\hline Agricultura en general & 631 & 73 & 90 & 57 \\
\hline
\end{tabular}




\begin{tabular}{|c|c|c|c|c|}
\hline Sector & CDU & 2003 & 2004 & 2005 \\
\hline Maquinaria & 631.3 & 45 & 60 & 37 \\
\hline Plantas de jardín. Jardinería & 635 & 28 & 30 & 20 \\
\hline Ganadería & 636 & 31 & 47 & 27 \\
\hline Animales & 636.04 & 9 & 15 & 11 \\
\hline Restaurantes y bares & 640.4 & 130 & 166 & 165 \\
\hline Mobiliario y accesorios & 645 & 95 & 112 & 75 \\
\hline Ferretería y menaje & {$[645+683.1]: 658.8$} & 19 & 21 & 15 \\
\hline Vestido. Cuidados corporales & 646 & 30 & 37 & 44 \\
\hline Cuidados corporales & 646.7 & 24 & 24 & 21 \\
\hline Organización de oficinas. Limpieza & $651: 648$ & 112 & 135 & 94 \\
\hline Mobiliario. Equipos. Máquinas de oficina. Material de oficina & 651.2 & 96 & 118 & 80 \\
\hline Telecomunicaciones & 654 & 18 & 25 & 15 \\
\hline Artes gráficas & 655 & 41 & 63 & 31 \\
\hline Publicidad y propaganda. Información & 659 & 106 & 192 & 69 \\
\hline Publicidad y marketing & 659.1 & 31 & 37 & 33 \\
\hline Imagen y sonido & $659.13 / .14$ & 25 & 31 & 36 \\
\hline Medios de comunicación & 659.3 & 50 & 124 & \\
\hline Empresas de expedición. Agencias de transportes & 659.96 & 7 & 7 & 7 \\
\hline Madera e industria de la madera & 674 & 118 & 53 & 28 \\
\hline Industria de los plásticos & 678 & 5 & 8 & 6 \\
\hline Mecanismos e instrumentos de precisión & 681 & 20 & 25 & \\
\hline Industria del calzado & 685.34 & 29 & 42 & 30 \\
\hline Tiendas de moda & 687 & 76 & 85 & 57 \\
\hline Juguetes & 688 & 6 & 13 & 13 \\
\hline Fabricantes & $62 / 69$ & 47 & 61 & 54 \\
\hline Materiales de construcción. Prácticas y procedimiento... & 69 & 277 & 266 & 201 \\
\hline Recursos energéticos en general & 690.91 & 20 & 19 & 17 \\
\hline Materiales de construcción & 691 & 80 & 96 & 75 \\
\hline Fontaneros. Electricistas. Otros oficios & 696 & 67 & 97 & 67 \\
\hline Calefacción, ventilación y acondicionamiento & 697 & 25 & 36 & 27 \\
\hline Arquitectura y diseño & 698 & 18 & 18 & 15 \\
\hline Artesanía & 7.02 & 42 & 62 & 37 \\
\hline Fotografía y procesos similares & 77 & 10 & 18 & 11 \\
\hline Diversiones. Espectáculos. Juegos. Deportes & 79 & 65 & 100 & 59 \\
\hline Espectáculos & 791 & 25 & 37 & 26 \\
\hline Deportes. Juegos deportivos & 796 & 40 & 63 & 33 \\
\hline
\end{tabular}

Tabla I. Páginas web comerciales recuperadas y clasificadas según la CDU durante los años 2003, 2004 y 2005.

Scire. $12: 1$ (en.-jun. 2006) 163-178. ISSN 1135-3761. 
Si observamos la tabla I vemos cómo ha ido evolucionando el comercio electrónico en Extremadura durante estos casi tres años; así, en general observamos que en este corto periodo de tiempo ha ido creciendo considerablemente el número de páginas web comerciales. Si bien es cierto que en algunos sectores presentan menores resultados en el año 2005, creemos que es posible que esto sea debido a aquellas empresas que finalmente no se adaptan a la Red y deciden abandonarla (ahora bien, hay que tener en cuenta que los datos del 2005 no son anuales sino semestrales, por lo que quizás sea mejor realizar una nueva búsqueda en octubre y comprobar esos datos).

Para ver más detenidamente la evolución del comercio electrónico extremeño mostramos las siguientes gráficas con los sectores con más representación en la Red. Hemos cogido los ocho primeros (en función del número de sitos web). Así, los resultados para el año 2003 se pueden ver en la gráfica 1 .

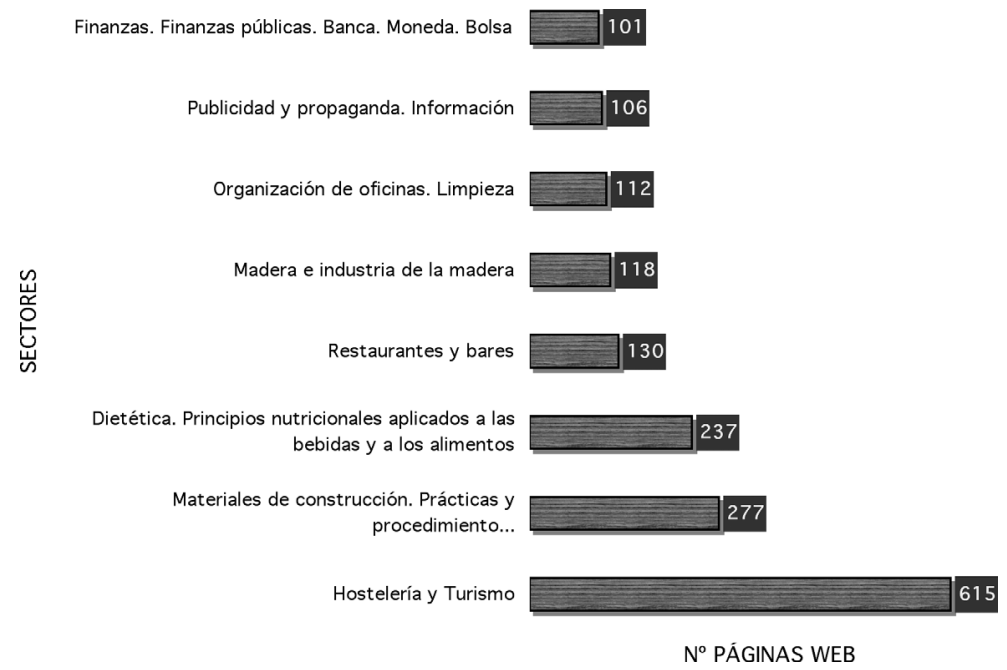

Gráfica 1. Sectores más representativos en la Red durante el año 2003 con el número de páginas web recuperadas

Queda muy claro que el sector más representativo en Extremadura es el de hostelería y turismo, donde tiene un papel muy importante el turismo rural.

La gráfica 2 muestra los ocho primeros sectores, pero del año 2004.

En este año la hostelería sigue siendo el primer sector, pero destaca que el segundo lugar pasa a ocuparlo dietética y principios nutricionales, que representa al sector de la alimentación extremeño, en detrimento de materiales de construcción.

Scire. $12: 1$ (en.-jun. 2006) 163-178. ISSN 1135-3761. 


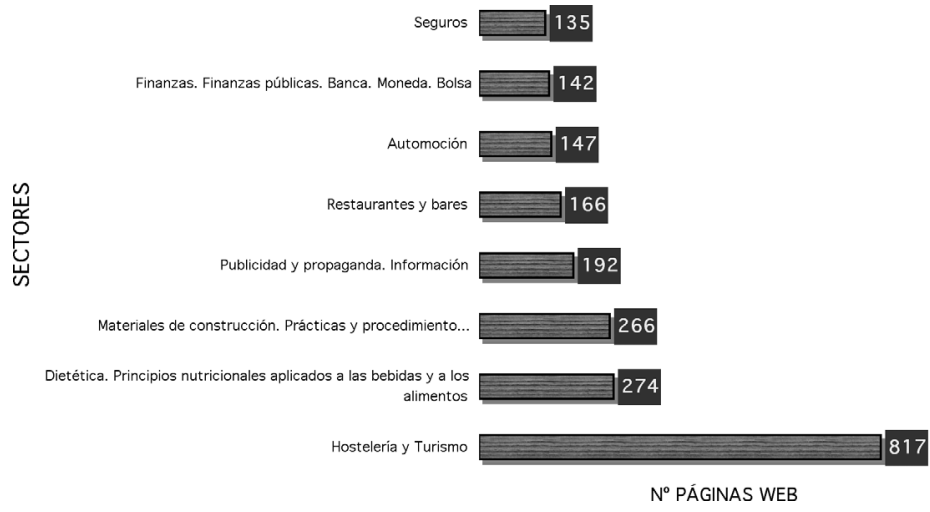

Gráfica 2. Sectores más representativos en la Red durante el año 2004 con el número de páginas web recuperadas

Finalmente, la gráfica 3 muestra los ocho primeros sectores del año 2005.

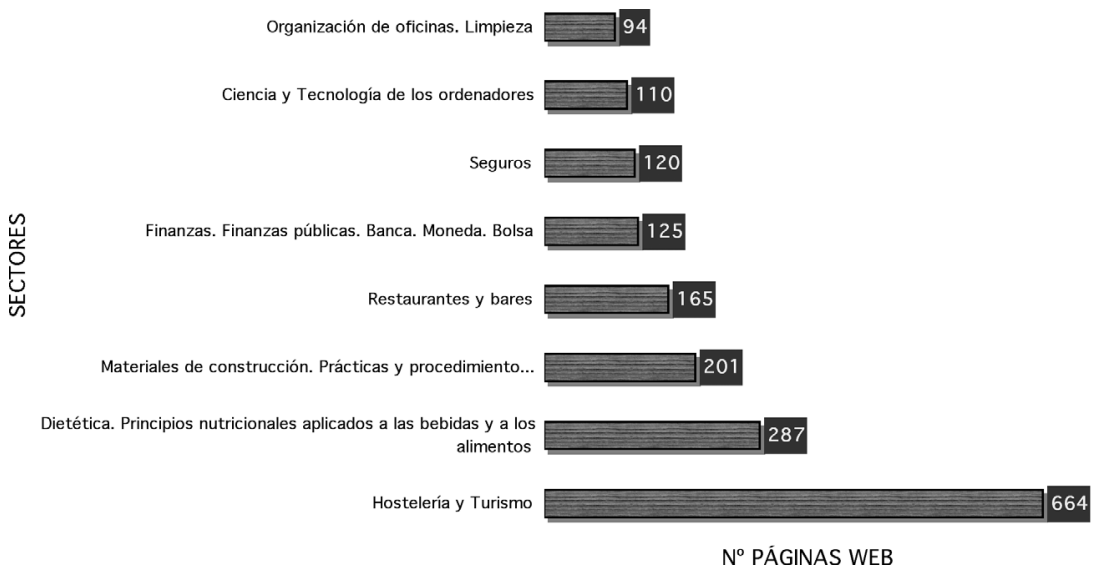

Gráfica 3. Sectores más representativos en la Red durante el año 2005 con el número de páginas web recuperadas

La gráfica 3 indica que durante el último año, si bien los datos se mantienen muy parecidos a los de 2004, en el caso de 2005 el sector de publicidad y propaganda e información muestra una considerable disminución con respecto a los años anteriores, hasta desaparecer de entre los ocho primeros.

Scire. $12: 1$ (en.-jun. 2006) 163-178. ISSN 1135-3761. 
Finalmente, la gráfica 4 muestra una comparación entre los ocho primeros sectores del año 2003 junto con los datos de 2004 y 2005.

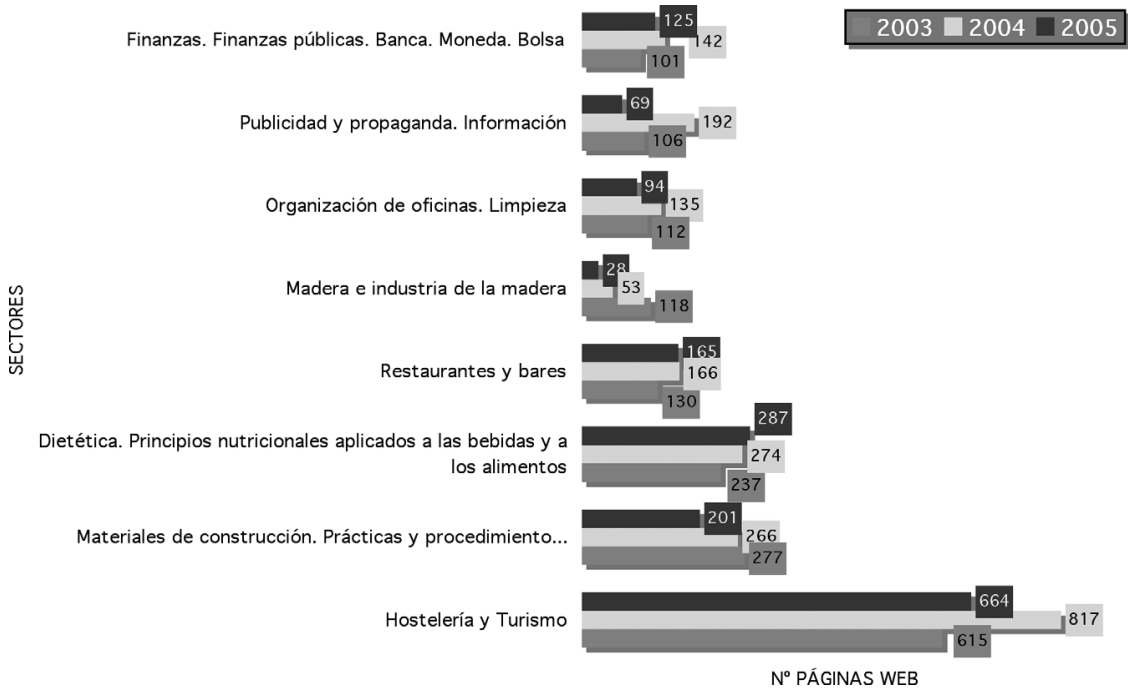

Gráfica 4. Comparación de los ocho sectores más representativos del año 2003 con los datos obtenidos durante 2004 y 2005

Esta gráfica muestra el año 2004 como el de máxima difusión del comercio electrónico en esta comunidad autónoma. Si bien en el año 2005 se presenta cierta disminución, creemos que todavía no hay que tenerla en cuenta, pues los datos cuatrimestrales que presenta la AECE a veces muestran esta bajadas temporales, que en ocasiones se recuperan en los siguientes cuatrimestres, por lo que creemos que lo prudente es esperar y realizar un nuevo recuento en octubre de 2005.

En la tabla II se muestran los datos, ordenados según el código de la CDU, correspondientes al número de páginas que presentan venta directa, es decir, aquellas en las que, además de mostrarnos las características de la empresa, situación, catálogo, etcétera, se nos permite comprar el producto sin movernos de casa. No siempre podemos obtener el producto a través del ordenador, aunque nosotros consideramos la venta como directa siempre que no haya que ir a la tienda, escribir un e-mail, llamar a un teléfono, es decir, que se pueda realizar la petición directamente desde la página web, aunque luego recibamos nuestra compra por correo. 


\begin{tabular}{|c|c|c|c|}
\hline & 2003 & 2004 & 2005 \\
\hline Lectura & & 3 & 5 \\
\hline Comercio del suelo. Comercio inmobiliario & & 4 & 4 \\
\hline Mercado de las viviendas de alquiler & & 7 & 7 \\
\hline Finanzas. Finanzas públicas. Banca. Moneda. Bolsa & & 20 & 18 \\
\hline Hospitales & & & 1 \\
\hline Seguros & & 15 & 15 \\
\hline Clases de empresas de seguros. Compañías mixtas & & 15 & 15 \\
\hline Educación. Enseñanzas. Formación & 5 & 3 & 2 \\
\hline Formación general (formación) + otros métodos de enseñanza (academias) & & 3 & 2 \\
\hline Hostelería y turismo & 2 & 177 & 177 \\
\hline Hostales y pensiones & & 16 & 16 \\
\hline Hoteles & & 72 & 72 \\
\hline Turismo rural & & 82 & 82 \\
\hline Camping & & 1 & 1 \\
\hline Viajes/agencias & & 6 & 6 \\
\hline Fiestas nacionales. Festejos populares & & 1 & 1 \\
\hline Ciencia y tecnología de los ordenadores & & 14 & 20 \\
\hline Protección del medio ambiente & & 1 & 1 \\
\hline \multicolumn{4}{|l|}{ Química } \\
\hline $\begin{array}{l}\text { Dietética. Principios nutricionales aplicados a las bebidas } \\
\text { y a los alimentos }\end{array}$ & 60 & 122 & 88 \\
\hline Jamones y embutidos & & 64 & 47 \\
\hline Bebidas y bodegas & & 35 & 19 \\
\hline Almazaras y aceitunas & & 10 & 9 \\
\hline Automoción & 3 & & 2 \\
\hline Taller & & & 1 \\
\hline \multicolumn{4}{|l|}{ Recambio } \\
\hline Ventas & & & 1 \\
\hline Higiene y salud pública & 2 & 3 & 1 \\
\hline Farmacia general y profesional & & 3 & 1 \\
\hline \multicolumn{4}{|l|}{ Ingenierías } \\
\hline \multicolumn{4}{|l|}{ Ingeniería eléctrica } \\
\hline \multicolumn{4}{|l|}{ Electrodomésticos } \\
\hline Agricultura en general & 2 & 2 & 3 \\
\hline \multicolumn{4}{|l|}{ Maquinaria } \\
\hline Plantas de jardín. Jardinería & & 1 & 1 \\
\hline Ganadería & & 1 & 2 \\
\hline Animales & & 1 & 1 \\
\hline Restaurantes y bares & & & \\
\hline
\end{tabular}

Scire. $12: 1$ (en.-jun. 2006) 163-178. ISSN 1135-3761. 


\begin{tabular}{|c|c|c|c|}
\hline & 2003 & 2004 & 2005 \\
\hline Mobiliario y accesorios & & 1 & 1 \\
\hline \multicolumn{4}{|l|}{ Ferretería y menaje } \\
\hline Vestido. Cuidados corporales & & 1 & 1 \\
\hline \multicolumn{4}{|l|}{ Cuidados corporales } \\
\hline Organización de oficinas. Limpieza & & 6 & 6 \\
\hline Mobiliario. Equipos. Máquinas de oficina. Material de oficina. & & 6 & 6 \\
\hline Telecomunicaciones & & 3 & 1 \\
\hline Artes gráficas & & 2 & 2 \\
\hline Publicidad y propaganda. Información & & 35 & 7 \\
\hline Publicidad y marketing & & 1 & 1 \\
\hline Imagen y sonido & & 12 & 6 \\
\hline Medios de comunicación & & 22 & \\
\hline Empresas de expedición. Agencias de transportes & & 6 & 6 \\
\hline \multicolumn{4}{|l|}{ Madera e industria de la madera } \\
\hline \multicolumn{4}{|l|}{ Industria de los plásticos } \\
\hline \multicolumn{4}{|l|}{ Mecanismos e instrumentos de precisión } \\
\hline Industria del calzado & & 2 & 2 \\
\hline \multicolumn{4}{|l|}{ Tiendas de moda } \\
\hline Juguetes & & 2 & 2 \\
\hline Fabricantes & & 2 & 2 \\
\hline Materiales de construcción. Prácticas y procedimiento... & & 7 & 7 \\
\hline \multicolumn{4}{|l|}{ Recursos energéticos en general } \\
\hline Materiales de construcción & & 3 & 3 \\
\hline Fontaneros. Electricistas. Otros oficios & & 3 & 3 \\
\hline \multicolumn{4}{|l|}{ Calefacción, ventilación y acondicionamiento } \\
\hline Arquitectura y diseño & & 1 & 1 \\
\hline Artesanía & & 3 & 3 \\
\hline \multicolumn{4}{|l|}{ Fotografía y procesos similares } \\
\hline Diversiones. Espectáculos. Juegos. Deportes & & & 3 \\
\hline \multicolumn{4}{|l|}{ Espectáculos } \\
\hline Deportes. Juegos deportivos & & & 3 \\
\hline
\end{tabular}

Tabla II. Muestra las páginas web comerciales recuperadas que presentan venta directa durante los años 2003, 2004 y 2005

Se puede observar en esta tabla que el sector de la alimentación y hostelería es el más representativo del comercio electrónico directo extremeño, destacando el hecho de que Extremadura es la única comunidad autónoma que presenta datos representativos del sector de la alimentación en la Red (Fernández y Peral, 2003). 
La tabla III muestra los resultados de venta directa, y se caracteriza porque en los datos correspondientes al año 2003 solo se muestran los sectores (negritas) y no los subsectores, lo mismo que en la tabla 2. Esto es debido a que, cuando se recogieron, al ser tan escaso el número, se decidió indicar únicamente los sectores en que se clasificaban, no incluyéndose los datos específicos de cada subsector. En cambio, en los años 2004 y 2005, como al recuperarlos utilizamos la CDU y además aumentó considerablemente el número de páginas web comerciales de venta directa, se ha especificado cada uno de ellos.

\begin{tabular}{|c|c|c|c|}
\hline & 2003 & 2004 & 2005 \\
\hline Lectura & 34 & 34 & 18 \\
\hline Comercio del suelo. Comercio inmobiliario & 56 & 70 & 58 \\
\hline Mercado de las viviendas de alquiler & 23 & 12 & 17 \\
\hline Finanzas. Finanzas públicas. Banca. Moneda. Bolsa & 101 & 122 & 107 \\
\hline Hospitales & & 7 & 4 \\
\hline Seguros & 78 & 120 & 105 \\
\hline Clases de empresas de seguros & & 104 & 87 \\
\hline Educación. Enseñanzas. Formación & 69 & 75 & 46 \\
\hline Formación general (formación) + otros métodos de enseñanza (academias) & & 75 & 46 \\
\hline Hostelería y turismo & 613 & 640 & 487 \\
\hline Hostales y pensiones & & 296 & 268 \\
\hline Hoteles & & 95 & 59 \\
\hline Turismo rural & & 184 & 107 \\
\hline Camping & & 32 & 25 \\
\hline Viajes/agencias & & 33 & 28 \\
\hline Fiestas nacionales. Festejos populares & 4 & 13 & 12 \\
\hline Ciencia y tecnología de los ordenadores & 10 & 46 & 90 \\
\hline Protección del medio ambiente & 8 & 9 & 9 \\
\hline Química & 9 & 7 & 6 \\
\hline $\begin{array}{l}\text { Dietética. Principios nutricionales aplicados a las bebidas } \\
\text { y a los alimentos }\end{array}$ & 177 & 152 & 199 \\
\hline Jamones y embutidos & & 6 & 23 \\
\hline Bebidas y bodegas & & 4 & 21 \\
\hline Almazaras y aceitunas & & 51 & 53 \\
\hline Automoción & 89 & 147 & 89 \\
\hline Taller & & 59 & 37 \\
\hline Recambio & & 27 & 13 \\
\hline Ventas & & 55 & 34 \\
\hline Higiene y salud pública & 50 & 69 & 71 \\
\hline Farmacia general y profesional & & 5 & 3 \\
\hline Ingenierías & 76 & 112 & 73 \\
\hline Ingeniería eléctrica & & 60 & 37 \\
\hline
\end{tabular}

Scire. $12: 1$ (en.-jun. 2006) 163-178. ISSN 1135-3761. 


\begin{tabular}{|c|c|c|c|}
\hline & 2003 & 2004 & 2005 \\
\hline Electrodomésticos & & 23 & 14 \\
\hline Agricultura en general & 71 & 88 & 54 \\
\hline Maquinaria & & 60 & 37 \\
\hline Plantas de jardín. Jardinería & & 29 & 19 \\
\hline Ganadería & 31 & 46 & 25 \\
\hline Animales & & 14 & 10 \\
\hline Restaurantes y bares & 130 & 166 & 165 \\
\hline Mobiliario y accesorios & 95 & 111 & 74 \\
\hline Ferretería y menaje & & 21 & 15 \\
\hline Vestido. Cuidados corporales & 30 & 36 & 43 \\
\hline Cuidados corporales & & 24 & 21 \\
\hline Organización de oficinas. Limpieza & 112 & 129 & 88 \\
\hline Mobiliario. Equipos. Máquinas de oficina. Material de oficinas & & 112 & 74 \\
\hline Telecomunicaciones & 18 & 22 & 14 \\
\hline Artes gráficas & 41 & 61 & 29 \\
\hline Publicidad y propaganda. Información & 106 & 157 & 62 \\
\hline Publicidad y marketing & & 36 & 32 \\
\hline Imagen y sonido & & 19 & 30 \\
\hline Medios de comunicación & & 102 & \\
\hline Empresas de expedición. Agencias de transportes & 7 & 1 & 1 \\
\hline Madera e industria de la madera & 118 & 53 & 28 \\
\hline Industria de los plásticos & 5 & 8 & 6 \\
\hline Mecanismos e instrumentos de precisión & 20 & 25 & \\
\hline Industria del calzado & 29 & 40 & 28 \\
\hline Tiendas de moda & 76 & 85 & 57 \\
\hline Juguetes & 6 & 11 & 11 \\
\hline Fabricantes & 47 & 59 & 52 \\
\hline Materiales de construcción. Prácticas y procedimiento... & 277 & 259 & 194 \\
\hline Recursos energéticos en general & & 19 & 17 \\
\hline Materiales de construcción & & 93 & 72 \\
\hline Fontaneros. Electricistas. Otros oficios & & 94 & 64 \\
\hline Calefacción, ventilación y acondicionamiento & & 36 & 27 \\
\hline Arquitectura y diseño & & 17 & 14 \\
\hline Artesanía & 42 & 59 & 34 \\
\hline Fotografía y procesos similares & 10 & 18 & 11 \\
\hline Diversiones. Espectáculos. Juegos. Deportes & 65 & 100 & 56 \\
\hline Espectáculos & & 37 & 26 \\
\hline Deportes. Juegos deportivos & & 63 & 30 \\
\hline
\end{tabular}

Tabla III. Muestra las páginas web comerciales recuperadas que presentan venta indirecta durante los años 2003, 2004 y 2005 
Al observar las tablas II y III se pone de manifiesto una serie de características propias del mercado electrónico extremeño: el empresario de esta comunidad se ha sumado con cierta rapidez a las nuevas tecnologías, y así se pone de manifiesto en el número de empresas recuperadas con venta indirecta (por tanto, la posibilidad de utilizar la Red como medio para publicitar y dar a conocer su empresas ha sido muy bien aceptada); en cambio, el caso de la venta directa, donde el empresario extremeño tiene que hacer una pequeña inversión y mantenimiento de la infraestructura creada para vender en Internet, sigue siendo la asignatura pendiente del sector empresarial extremeño y es donde hay que hacer un mayor esfuerzo, tanto desde el punto de vista institucional como desde el sector privado dedicado a la venta de estos productos, para que la comunidad empresarial extremeña se adapte plenamente al nuevo medio comercial que es Internet.

\section{Conclusiones}

El sector de la alimentación clasificado como "Dietética. Principios nutricionales aplicados a las bebidas y a los alimentos" es uno de los más representativos del comercio electrónico extremeño, a diferencia del nacional.

El sector de hostelería y turismo es el que más páginas web comerciales presenta en los tres años, e incluso en 2004 y 2005 es el que tiene el mayor número de sitios con venta directa.

Internet ha sido asumido por el sector empresarial extremeño como medio para dar a conocer sus empresas, pero no como medio para realizar transacciones comerciales.

\section{Referencias}

AECE, Asociación Española de Comercio Electrónico (2003). Estudio de comercio electrónico B2C en España. AECE-fecemd 2003. URL: <http://www.aece.org/noticias.asp>. Consultado: 2005-03-13.

Andersen Consulting (1999). La empresa española ante el comercio electrónico, 1999.

Andersen Consulting España; Banco Santander Central Hispano (2000). España on line: ideas para afrontar la e-economía, 2000. URL: <http://ciberconta.unizar.es/ftp/pub/docs/ bsch/online.html>. Consultado: 2004-10-06.

Caro Castro, C. (1998). Sistemas de clasificación y organización de la información en Internet. // Fesabid 98: VI Jornadas Españolas de Documentación, 1998. URL: <http://fesabid98.florida-uni.es/Comunicaciones/c_caro.htm>. Consultado: 2004-03-14.

eMarket Services. Your guide to electronic market places (2005). URL: <www.emarket services.com>. Consultado: 2005-05-18.

Fernández Falero, M. R.; Peral Pacheco, D. (2003). Situación del comercio electrónico en Extremadura después de la crisis de Internet. // CALSI 2003: Contenidos y aspectos legales en la sociedad de la información. CD-Rom. Valencia: Universidad Politécnica, 2003.

Scire. $12: 1$ (en.-jun. 2006) 163-178. ISSN 1135-3761. 
Giorno, C.; Jiménez, M. (2005). Estudio económico de España, 2005. ECO/EDR 2005, $\mathrm{n}^{\circ}$ 2. URL: <www.oedd.org/dataoecd>. Consultado: 2005-03-16.

Extremadura Empresas. URL: <www.extremaduraempresas.com>. Consultado: 2005-03-03.

Más Empresa. Portal de empresas y profesionales. URL: <www.masempresa.com>. Consultado: 2005-03-03.

Potts, R. F. (2004). Comercio electrónico: herramientas para los negocios del tercer milenio. URL: <http://www.gestiopolis.com/recursos/documentos/fulldocs/ger1/ comleectro.htm>. Consultado: 2004-09-07.

Santamaría Díaz, F.; Escobar Espinar, M. (2004). Estrategias empresariales ante el comercio electrónico. // ICE. El Comercio en la Sociedad de la Información. 813 (febrero 2004) 187-196.

Solé Moro, M. L. (2000). Comercio electrónico: un mercado en expansión. Madrid: ESIC, 2000.

Vizuete Gómez, M. (2001). Marketing y comercio electrónico. URL: <http://www.marketing ycomercio.com/numero5/posicionamiento.htm>. Consultado: 2004-09-07. 